\title{
Drug-, herb- and dietary supplement-induced liver injury
}

\author{
María L. Cavalieri, M.D. ${ }^{a}$ and Daniel D'Agostino, M.D. ${ }^{a}$
}

\begin{abstract}
Drug- and substance-induced liver injury accounts for approximately $20 \%$ of pediatric cases of acute liver failure. It is caused by two mechanisms: direct and idiosyncratic hepatotoxicity.

Direct hepatotoxicity is the result of the administration of a drug with intrinsic toxicity and is dose-dependent (e.g., acetaminophen). Idiosyncratic hepatotoxicity is unpredictable, uncommon, variable in presentation, and doseindependent.

The clinical, histological, and laboratory manifestations include hepatitis, which is generally asymptomatic but with a significant increaseofliverenzymes; cholestasis, accompanied with jaundice, pruritus, prominent elevation of alkaline phosphatase, and mild elevation of aminotransferases; or mixed, with elements of both hepatitis and cholestasis.

Time to recovery is variable, depending on the type of liver injury. Early detection and discontinuation of the causative drug is the most effective and important step for the fast resolution of histological and clinical changes, thus reducing severe liver injury.

Key words: Drug-induced liver injury.
\end{abstract}

http:/ / dx.doi.org/10.5546/aap.2017.eng.e397

To cite: Cavalieri ML, D'Agostino D. Drug-, Herb- and Dietary Supplement-Induced Liver Injury. Arch Argent Pediatr 2017;115(6):e397-e403.

a. Department of Pediatric Gastroenterology, Hepatology, and Liver and Intestinal Transplant of Hospital Italiano de Buenos Aires.

E-mail address:

María L. Cavalieri, M.D.: maria.cavalieri@ hospitalitaliano.org.ar.

Funding:

None.

Conflict of interest: None.

Received: 2-21-2017 Accepted: 6-8-2017

\section{INTRODUCTION}

Drug- and substance-induced hepatotoxicity, known internationally as drug-induced liver injury (DILI), is a relevant entity in everyday clinical practice. The incidence of DILI is unknown because few prospective population studies have been conducted. The annual incidence of DILI in the general population has been estimated to be 1-2 cases per 100000 individuals.

In Western countries, most DILI cases are associated with antibiotics, anticonvulsant agents, and psychotropic agents. In Asian countries, herbs and dietary supplements are the most common causes of DILI. ${ }^{1}$

In the United States of America and Europe, intrinsic hepatotoxicity accounts for $7-15 \%$ of acute liver failure cases and is the most common cause of discontinuation of a marketapproved drug. ${ }^{2}$

In the field of pediatrics, druginduced hepatotoxicity accounts for $20 \%$ of acute liver failure cases, $14 \%$ of which have been reported in association with acetaminophen.

At present, more than 1000 drugs and substances have been reported to cause hepatotoxicity among children and adults. ${ }^{3,4}$ The wide range of clinical manifestations, the multiple number of causative agents, and the lack of specific diagnostic tests make diagnosis even much more complicated. In spite of its low incidence in the general population, DILI should always be suspected in patients with idiopathic acute liver disease. Many herbal and dietary supplements can cause DILI and should therefore be considered if liver injury is suspected. Genetic and environmental factors, in association with drug exposure, determine the development of hepatotoxicity in each child and adolescent.

\section{DRUG SUSCEPTIBILITY}

Drug biotransformation in the liver starts in the endoplasmic reticulum with cytochrome P450 oxidation followed by $\mathrm{N}$-acetyltransferase and glutathione transferase conjugation, which produces metabolites, called haptens, that are later bound to hepatocyte proteins. Haptens are presented to the human leukocyte antigen (HLA), thus generating an innate immune response which, in most cases, resolves with immune tolerance. 
Given that HLA molecules are highly polymorphic and participate in specific immune system activation, some allele and haplotype variants may be related to hepatotoxic immune reactions. ${ }^{5}$ This way, the genetic polymorphism makes certain drugs or substances to have a different risk of susceptibility to toxicity. ${ }^{6}$

Therefore, not all individuals react in the same way when taking the same drug or substance. Some people are tolerant: liver injury is undetectable; others are adapters: liver injury is transient and selective, and liver function is normalized, even if the drug administration continues; and others are susceptible: liver injury is clinically significant and may revert on drug discontinuation. ${ }^{7}$

A useful and common manner to characterize hepatotoxicity is to classify it into intrinsic or idiosyncratic. Intrinsic hepatotoxicity affects all individuals to a varying degree and is dosedependent. Acetaminophen is one of the causative drugs. The efficacy and safety of acetaminophen in children have been adequately established; however, toxicity has increased over the last years. The toxic dose is $120-150 \mathrm{mg} / \mathrm{kg}$, and continuous doses establish a "memory" response.

Idiosyncratic hepatotoxicity is defined as an unexpected adverse reaction that affects only susceptible individuals, is not as dose-dependent, and varies with latency, manifestation, and course. ${ }^{8,9}$

Risk factors for DILI are age, sex, nutritional status, concomitant drug use, tobacco use, and genetics. Chronic liver diseases, such as hepatitis C, fatty liver, and alcohol use, are predisposing factors for a greater susceptibility to certain drugs or substances. ${ }^{1,10}$

\section{CLINICAL MANIFESTATIONS AND DIAGNOSIS}

DILI signs, symptoms, and laboratory findings may mimic any clinical presentation of acute or chronic liver disease. There are no specific hepatotoxicity laboratory findings, except acetaminophen dosage when this is the causative drug. The diagnosis is one of exclusion, based on the clinical judgment and the knowledge about the potential causative agent of liver injury.

However, there are biochemistry criteria defining this type of DILI:

- Serum alanine aminotransferase (ALT) 5 times higher than normal.

- Serum alkaline phosphatase (AP) 2 times higher than normal.

- Serum ALT 3 times higher than normal together with serum bilirubin 2 times higher than normal. ${ }^{2}$

To assess the probability of DILI, the following aspects should be considered:

- Latency: It represents the time from treatment initiation with a drug or substance until the onset of liver injury, determined either clinically or through abnormal liver function tests. This interval should be between 5 and 90 days to be considered strongly associated with hepatotoxicity. Latency is classified into short (3-30 days), moderate (30-90 days) or long (more than 90 days). Exceptionally, latency may be 24-72 hours, in general, due to drug rechallenge (sulfonamides and macrolids), 3-12 months (isoniazid) or more than a year (nitrofurantoin, cyproterone). ${ }^{9,11}$

- Time to recovery: It represents the time from drug discontinuation until full recovery from the liver injury, with liver function test values equal to those at the onset of hepatotoxicity. It is estimated that improvement occurs after 1-2 weeks of drug discontinuation and recovery, after 2-3 months.

The adjudication of causality to suspected DILI was assessed using the Roussel Uclaf Causality Assessment Method (RUCAM), which classifies liver injury as having a hepatocellular, mixed or cholestatic pattern. The score ranges from -9 to +10 , with higher scores indicating a higher likelihood of DILI. Duration of drug exposure, liver function test values, risk factors, concomitant medications, differential diagnoses, and information following drug rechallenge are assessed. ${ }^{1,9,12,13}$

\section{CLINICAL, HISTOLOGICAL, AND LABORATORY MANIFESTATIONS}

Biochemical abnormalities and histological characteristics, in association with clinical presentation, help to define the pattern of a drugor substance-induced liver injury. Risk $(R)$ is used, defined as a serum ALT value above normal and divided into the serum AP value above normal. If $R>5$, the pattern is defined as hepatocellular; $R=2-5$, mixed; $R<2$, cholestatic. $^{2}$

\section{Classification of liver injury based on liver function test abnormalities and histology:}

1. Hepatitis: $R>5$. Elevated ALT or ALT 3 times higher than normal, elevated aspartate aminotransferase (AST), mildly elevated PA and gamma-glutamyl transpeptidase (GGT). Symptoms: weakness, fatigue, vomiting. Histology: cell necrosis, inflammation, 
and moderate biliary stasis. Examples: acetaminophen, isoniazid, green tea.

2. Cholestasis: $R<2$. Elevated PA or PA 2 times higher than normal, very elevated GGT, mildly elevated ALT and AST. Symptoms: jaundice, pruritus, and right upper quadrant pain. Histology: biliary stasis, portal inflammation, ductal and ductular proliferation. Examples: amoxicillin/clavulanic acid, diphenylhydantoin, steroids.

3. Mixed: $R=2-5$. Similarly elevated ALT and PA. Symptoms: fatigue, jaundice, and pruritus. Histology: hepatocyte necrosis, inflammation, and marked biliary stasis. Examples: sulfonamides, diclofenac. ${ }^{14,15}$

Based on this classification, DILI may have the following clinical presentations (Table 1):

1. Acute hepatic necrosis: rapid onset of hepatocellular injury following drug exposure, with clinical manifestations and lab tests indicative of liver failure and marked ALT elevation. It may be fatal, but recovery of nonfatal cases is fast once the drug is discontinued. Examples: acetaminophen, aspirin.

2. Acute hepatitis: similar to viral acute hepatitis, with a marked elevation of ALT and hepatocellular injury. Symptoms include nausea, fatigue, jaundice, choluria, abdominal pain. Examples: isoniazid, ketoconazole.

3. Silent elevation of liver enzymes: the most common clinical manifestation, with elevated ALT and PA, and without clinical symptoms.
4. Chronic hepatitis: liver injury lasts for more than 6 months since drug discontinuation. In some cases, it only accounts for a slow improvement of liver injury; for example, the drug may trigger a chronic liver process, such as autoimmune hepatitis.

5. Sinusoidal obstruction syndrome: a rare but severe form that may cause acute liver failure. It is mainly observed among patients with bone marrow transplant. The most common symptoms include abdominal pain, edema, ascites. It is accompanied by elevated ALT and a hepatocellular pattern. Examples: chemotherapy and radiation.

6. Hepatic tumors: associated with longterm therapy and chronic liver injury. Examples: adenomas, with high dose estrogens; adenocarcinoma, with chronic use of androgens; and angiosarcoma, with radiological contrast media use.

7. Autoimmune hepatitis: it may present with increased immunoglobulin levels and positive markers of autoimmunity. Once the drug is discontinued, symptoms improve and lab tests return to normal. Examples: nitrofurantoin, methyldopa.

8. Nonalcoholic fatty liver: moderate and persistent elevation of liver enzymes with a hepatocellular pattern. In general, it is asymptomatic, diagnosed with a liver biopsy or ultrasound, and its latency is between 3 and 12 months. This condition resolves once the drug is discontinued. Examples: corticosteroids, antidepressants, antipsychotics, methotrexate. ${ }^{16}$

TABLE 1. Drugs and substances that most commonly cause hepatotoxicity

\begin{tabular}{|c|c|c|}
\hline Drug or substance & Latency* & Liver injury \\
\hline Amoxicillin/clavulanic acid & Short to moderate & Cholestasis \\
\hline Isoniazid & Moderate to long & Acute hepatitis / acute liver failure \\
\hline Pyrazinamide & Moderate & Hepatocellular \\
\hline Rifampicin & Short to moderate & Cholestasis \\
\hline Trimethoprim-sulfamethoxazole & Short to moderate & Cholestasis \\
\hline Nitrofurantoin & Moderate to long & Hepatocellular, autoimmune hepatitis \\
\hline Valproic acid & Moderate to long & Hepatocellular, hyperammonemia, microvesicular steatosis \\
\hline Carbamazepine & Moderate & Mixed, immune-allergic pattern, hypersensitivity syndrome \\
\hline Phenytoin & Moderate to long & Mixed, immune-allergic pattern, hypersensitivity syndrome \\
\hline Acetaminophen & Short to moderate & With high doses, hepatocellular injury/acute liver failure \\
\hline Diclofenac & Moderate to long & Hepatocellular \\
\hline Green tea & Short to moderate & Hepatocellular \\
\hline Anabolic steroids & Moderate to long & Cholestasis \\
\hline
\end{tabular}

* Short: 3-30 days; moderate: 30-90 days; long > 90 days. 


\section{Classification of severity in drug- and substance-induced liver injury}

The severity of liver injury is classified as mild, moderate or severe, depending on bilirubin and liver enzyme levels, signs of liver failure, recovery, chronic nature, or death (Table 2). ${ }^{1,2}$

\section{EXCLUSION OF OTHER DIAGNOSES}

The last step in DILI diagnosis is to rule out other conditions that may be affecting the liver. This includes obtaining a complete medical history, including personal history and concomitant drug use, lab tests, including liver function tests, coagulation profile, serology, markers of autoimmunity, protein profile, immunoglobulin level, serum copper, ceruloplasmin, 24-hour urine copper, and liver Doppler scan.

\section{When should a liver biopsy be done?}

1. Before indicating immunosupressors in the case of suspicion of autoimmune hepatitis versus DILI.

2. Increased liver enzymes or worsening of liver function after the discontinuation of the drug suspected to be responsible of DILI.

3. ALT levels that do not fall $>50 \%$ after 3060 days of onset (hepatocellular DILI) or PA levels that do not fall $>50 \%$ after 180 days of onset (cholestatic DILI), in spite of having discontinued the drug suspected to be responsible of DILI.

4. After 180 days of liver injury to assess chronic liver disease. ${ }^{16}$

\section{HEPATOTOXICITY AND HERBS, DIETARY SUPPLEMENTS, AND RECREATIONAL DRUGS}

The use of medicinal herbs has become increasingly common based, essentially, on the belief that natural products or herbs are always safe. Severe acute liver failure cases have been reported following the use of these products to treat different conditions. In the United States, the incidence is up to $9 \%$ whereas in Asian countries, it accounts for $19-63 \%$ of all DILI cases.

Herbs and dietary supplements more commonly associated with DILI include aloe vera, Asiatic pennywort, Herbalife ${ }^{\circledR}$, valerian, green tea, and OxyElite Pro ${ }^{\circledR}$, among others. ${ }^{17}$

Amanita phalloides is a fungus that grows in humid places, covered with dry leaves under oaks and pines. Its intake and subsequent poisoning causes an initial phase of gastrointestinal symptoms that may progress to liver failure, with a high mortality rate. The specific treatment is hemodynamic support and intravenous penicillin and silibinin with its isomer silymarin in the first 72 hours, both of which block amatoxin uptake by hepatocytes. In the case of children with acute liver failure, liver transplant should be considered early. In pathology studies, centrilobular necrosis and fatty degeneration are observed (Figure 1). ${ }^{18,19}$

Over the past years, the use of alcohol, recreational drugs, and anabolics has increased, mainly among adolescents.

Excessive alcohol use causes liver disease, including fatty liver, hepatitis, and cirrhosis. Alcohol is metabolized by the liver, and alcohol users would have an elevation predominantly of GGT and AST. Daily alcohol use should not exceed 14 grams in women and 28 grams in men. ${ }^{20,21}$

Cannabis or marijuana is the most common recreational drug worldwide. It is inhaled or used orally, as in foods or drinks. The most widely studied ingredients of marijuana are tetrahydrocannabinol and cannabidiol. In the liver, it is involved in drug metabolism via the cytochrome P450 and increases serum levels. In addition, cannabinoid receptor type 1 has steatogenic effects. Patients with

TABLE 2. Classification of severity of drug-induced liver injury

\begin{tabular}{lcr}
\hline Score & Severity & Definition \\
\hline 1 & Mild & Elevated ALT and $/$ or PA but bilirubin $<2.5 \mathrm{mg} / \mathrm{dL}$ and INR $<1.5$ \\
2 & Moderate & Elevated ALT and $/$ or PA and bilirubin $>$ or $=2.5 \mathrm{mg} / \mathrm{dL}$ or INR $>$ or $=1.5$ \\
3 & Moderate-severe & Elevated ALT, PA and bilirubin or INR, with hospitalization for DILI event \\
4 & Severe & Elevated ALT and / or PA and bilirubin $>$ or $=2.5 \mathrm{mg} / \mathrm{dL}$, with one of the following: \\
& - Liver failure (INR $>$ or $=1.5$, ascites or encephalopathy) \\
5 & Fatal & - Other organ failure caused by DILI (kidney or lung) \\
& Liver transplant or death caused by DILI event
\end{tabular}

ALT: alanine aminotransferase; PA: alkaline phosphatase; INR: international normalized ratio; DILI: drug-induced liver injury. 
chronic hepatitis $C$ usually have hepatic steatosis; therefore, daily cannabis use may worsen their steatosis and accelerate progression to fibrosis. ${ }^{22}$

Cocaine is a drug of abuse that is used intranasally, intravenously or inhaled. It is considered the most dangerous illegal drug. It causes cytolytic hepatitis, a significant elevation of ALT few hours after use, in association with rhabdomyolysis and acute renal failure. It may be accompanied by shock, hyperthermia, and hypotension. ${ }^{23}$

\section{RECHALLENGE WITH A HEPATOTOXIC DRUG}

Rechallenge with a drug suspected to have caused hepatotoxicity is not recommended, especially in the case of an initial liver injury with a significant ALT elevation, liver failure or prolonged jaundice. An exception may be made when the patient's life depends on such drug

FIGURE 1. Explanted liver, gross examination and histology from a patient with acute liver failure caused by Amanita phalloides poisoning

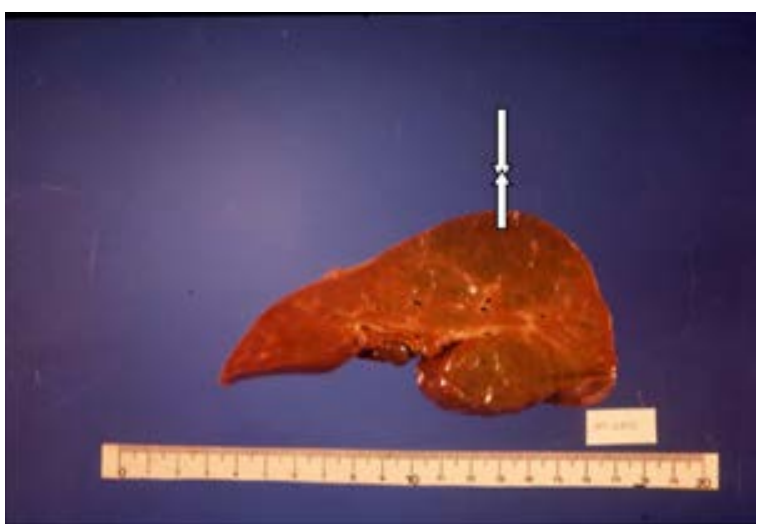

Gross examination of explanted liver: areas of hemorrhagic necrosis

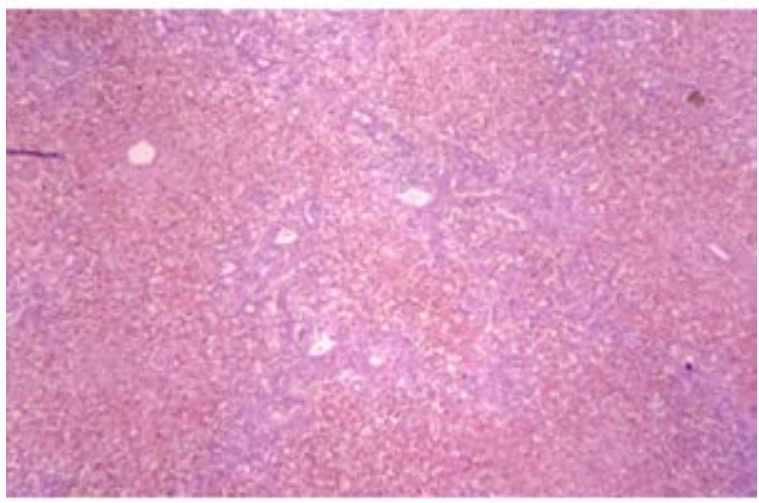

Histology: submassive and centrilobular hemorrhagic necrosis. or there is no alternative treatment. In this case, rechallenge should be progressive and under strict monitoring of liver function tests.

\section{TREATMENT}

Early detection and discontinuation of the hepatotoxic drug prevent liver injury progression. Treatment of liver failure caused by hepatotoxicity is similar to that caused by a virus, and may even require liver transplant. There are two prominent drugs for a specific treatment: $\mathrm{N}$-acetylcysteine for acetaminophenrelated toxicity and carnitine for valproic acidrelated toxicity.

The use of ursodeoxycholic acid and antioxidants has been described for severe and prolonged cases of hepatotoxicity. Some studies have supported corticosteroid use in severe liver injury cases accompanied by a multisystem hypersensitivity reaction to reduce mortality. ${ }^{24,25}$

\section{COMMENT}

Liver injury induced by drugs, herbs, dietary supplements or products to enhance athletic performance is nowadays a major problem affecting the general population and patients with underlying conditions, and also falls within the scope of physicians, who are responsible for indicating different treatments, the pharmaceutical industry, and regulatory agencies.

DILI accounts for $10-20 \%$ of cases of acute liver failure and liver transplant. However, most information about hepatotoxicity is obtained from data provided by the reporting system and publications in scientific journals. Therefore, the actual quantification is quite likely only the "tip of the iceberg."

Although epidemiological information about the toxic effect of drugs in the liver is scarce, it is known that DILI incidence has increased along with the release of new drugs to the market, a longer life expectancy, use of multiple medications, and the more and more extended use of herbal products.

The diagnosis of hepatotoxicity is difficult and may require a long time to do tests and rule out other conditions.

If hepatotoxicity is suspected, it is important for pediatric clinicians to consult with pediatric hepatologists or toxicology centers. In Argentina, some referral toxicology centers, such as Hospital Posadas and Hospital de Niños Ricardo Gutiérrez, provide comprehensive information both in person and on the telephone. 
Given the importance of this topic, several Argentinean hepatologists are part of the study protocol of drug-associated liver disease for the Latin American DILI Registry, coordinated by the Department of Gastroenterology and Hepatology of Hospital Provincial del Centenario, in Rosario, and by the Division of Hepatology and Clinical Pharmacology of the Department of Digestive Tract Conditions of the hospital of the University of Malaga.

The Department of Pediatric Gastroenterology, Hepatology, and Liver and Intestinal Transplant of Hospital Italiano has a division of hepatotoxicity to provide guidance for physicians and patients about potentially hepatotoxic drugs and substances for the prevention of adverse events. In addition, they offer follow-up and, eventually, therapy to patients with DILI.

\section{Acknowledgments}

We would like to thank Fernando Bessone, M.D. and coordinator of the Latin American DILI Registry (LATINDILI), for his advice and support and for reviewing the manuscript.

\section{REFERENCES}

1. Fontana R, Seeff L, Andrade R, et al. Standardization of Nomenclature and Causality Assessment in Drug-Induced Liver Injury: Summary of a Clinical Research Workshop. Hepatology 2010;52(2):730-42.

2. Aithal G, Watkins P, Andrade R, et al. Case Definition and Phenotype Standardization in Drug-Induced Liver Injury. Clin Pharmacol Ther2011;89(6):806-15.

3. Molleston J, Fontana R, Lopez M, et al. Characteristics of Idiosyncratic Drug-induced Liver Injury in Children: Results From the DILIN Prospective Study. J Pediatr Gastroenterol Nutr 2011;53(2):182-9.

4. Amin M, Harpavat S, Leung D. Drug-induced liver injury in children. Curr Opin Pediatr 2015;27(5):625-33.

5. Ocete-Hita E, Salmerón-Fernández MJ,Urrutia-Maldonado $\mathrm{E}$, et al. Analysis of immunogenetic factors in idiosyncratic drug-induced liver injury in the paediatric population. J Pediatr Gastroenterol Nutr 2017;64(5):742-7.

6. Chang Cand Schiano T. Review article: drughepatotoxicity. Aliment Pharmacol Ther 2007;25(10):1135-51.

7. Pauls L, Senior J. Drug-Induced liver injury. Clinical investigator training course. 15 November 2012. [Online]. [Accessed on: May 26 $\left.{ }^{\text {th }}, 2016\right]$. Available at: https://www.fda.gov/downloads/training/ clinicalinvestigatortrainingcourse/ucm340019.pdf

8. American Academy of Pediatrics. Committee on Drugs. Acetaminophen toxicity in children. Pediatrics 2001;108(4):1020-4.

9. Chalasani N, Hayashi P, Bonkovsky H, et al. ACG
Clinical Guideline: The Diagnosis and Management of Idiosyncratic Drug-Induced Liver Injury. Am J Gatroenterol 2014;109(7):950-66.

10. Chalasani N, Fontana R, Bonkovsky H, et al. Causes, clinical features, and outcomes from a prospective study of druginduced liver injury in the United States. Gastroenterology 2008;135(6):1924-34.e1-4.

11. Bessone F, Lucena M, Roma M, et al. Cyproterone acetate induces a wide spectrum of acute liver damage including corticosteroid-responsive hepatitis: report of 22 cases. Liver Int 2016;36(2):302-10.

12. Benichou C, Danan G, Flahault A. Causality assessment of adverse reactions to drugs-II. An original model for validation of drug causality assessment methods: Case reports with positive rechallenge. J Clin Epidemiol 1993;46(11):1331-6.

13. Danan G, Benichou C. Causality assessment of adverse reactions to drugs-I. A novel method based on the conclusions of international consensus meetings: application to drug-induced liver injuries. J Clin Epidemiol1993;46(11):1323-30.

14. Teschke R, Wolff A, Frenzel C, et al. Drug and herb induced liver injury: Council for International Organizations of Medical Sciences scale for causality assessment. World J Hepatol 2014;6(1):17-32.

15. Saxena R. Liver Injury Due to Drugs and Herbal Agents. In: Saxena R. Practical Hepatic Pathology: A diagnostic approach. Philadelphia: Elsevier-Saunders; 2011: 311-51.

16. National Institute of Diabetes and Digestive and Kidney Diseases, National Library of Medicine. Liver Tox Database: Clinical and Research Information on Drug-Induced Liver Injury [Online]. [Accessed on: May 2016]. Available at: https: / / livertox.nlm.nih.gov.

17. Leise M, Poterucha J, Talwalkar J. Drug-induced liver injury. Mayo Clin Proc 2014;89(1):95-106.

18. Grabhorn E, Nielsen D, Hillebrand G, et al. Successful outcome of severe Amanita phalloides poisoning in children. Pediatr Transplant 2013;17(6):550-5.

19. Talamoni M, Cabrerizo S, Cari C, et al. Intoxicación por Amanita phalloides, diagnóstico y tratamiento. Arch Argent Pediatr2006;104(4):372-4.

20. Agarwal S, Fulgoni V, Lieberman H. Assessing alcohol intake \& its dose dependent effects on liver enzymes by 24-h recall and questionnaire using NHANES 2001-2010 data. Nutr J 2016;15(1):62.

21. Office of Disease Prevention and Health Promotion. Dietary Guidelines for Americans 2015-2020 [Online]. [Accessed on: December 2016]. Available at: https://health.gov/ dietaryguidelines / 2015/.

22. Mayo Clinic. Drugs and supplements. 2013 [Online]. [Accessed on: December 2016]. Available at: http:/ / www. mayoclinic.org/drugs-supplements.

23. Tarantino G, Citro V, Finelli C. Recreational Drugs: a New Health Hazard for Patients with Concomitant Chronic Liver Diseases. J Gastrointestin Liver Dis2014;23(1):79-84.

24. Murray K. Drug-Induced LiverDisease. In:Kelly D. Diseases of the Liver and Biliary System in Children. 3rd ed. United Kingdom: Blackwell Publishing; 2008:207-30.

25. Roberts E. Drug-Induced Liver Disease. In Suchy F, Sokol R,Balistreri W (edit). Liver Disease in Children. 3rd ed. Cambridge: Cambridge University Press; 2007:478-512. 